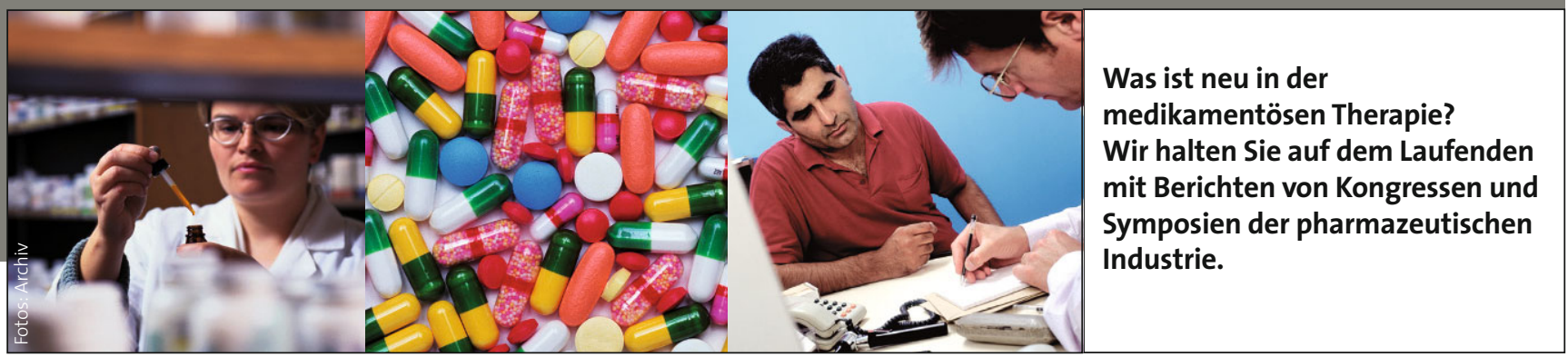

Neue Wege zum Therapieerfolg bei Morbus Bechterew

\title{
Monatsspritze und „Nur für mich“-Programm
}

- Patienten mit Morbus Bechterew (Spondylitis ankylosans, AS) lassen sich mit dem seit Kurzem verfügbaren TNF-alfa-Blocker Golimumab (Simponi ${ }^{\circledR}$ ) auch in der hausärztlichen Praxis deutlich einfacher führen. Der TNF-alfa-Blocker wird einmal monatlich subkutan appliziert. Bei der Patientenführung hilft das Programm „Just for me“, das der Hersteller Essex Pharma in Kooperation mit der Deutschen Vereinigung Morbus Bechterew e. V. entwickelt hat.

Das Angebot umfasst verschiedene Informations- und Beratungsmodule wie eine Patientenbroschüre, Internetseite, kostenfreie Telefonhotline und einen Erinnerungsdienst für die nächste Injektion und kann in teilnehmenden Praxen vom Patienten ausgewählt werden. In die Entwicklung dieses Programms sind die Erkenntnisse aus der RAISE-Studie eingeflossen. Diese internationale Patienten-

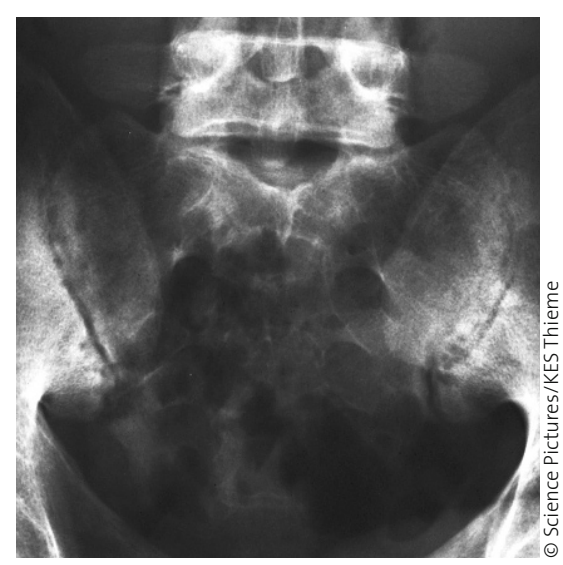

Frühzeitige AS-Diagnose verbessert die Behandlungsaussichten. befragung zeigte nicht nur die Wirksamkeit von Biologika bei chronisch entzündlichen Erkrankungen aus Sicht der Patienten, sondern auch die Wichtigkeit, die Patientenbedürfnisse bei der Therapiewahl mit zu berücksichtigen.

\section{Zeit bis zur Diagnose verkürzen}

Wie Prof. Herbert Kellner, München, betonte, ist eine frühe Diagnosestellung bei AS-Patienten deshalb so wichtig, weil eine rechtzeitig einsetzende effektive Therapie den Krankheitsverlauf maßgeblich günstig beeinflusst. Erst jüngst konnte eine randomisierte placebokontrollierte Studie an 40 Patienten mit sehr früher (<3 Jahre dauernder) axialer Manifestation einer AS den signifikanten Nutzen einer zwölfwöchigen Gabe von Infliximab (Remicade ${ }^{\circledR}$ ) belegen. Die klinischen und MRT-Befunde zeigten bereits nach dieser kurzen Behandlungszeit eine Reduktion der Krankheitsaktivität und eine Rückbildung der entzündlichen Läsionen.

Auch in der Zulassungsstudie für Golimumab (GO-RAISE) mit 356 AS-Patienten wurde ein bis zu zwei Jahre anhaltender Effekt einer monatlichen Gabe des TNFalfa-Blockers dokumentiert. Dieser Effekt umfasste eine signifikante Verbesserung der Krankheitsaktivität, der Wirbelsäulenfunktion und der Lebensqualität. Golimumab hat seit Oktober 2009 die Zulassung für AS sowie für rheumatoide Arthritis und Psoriasis-Arthritis.

\section{- Dr. Till U. Keil}

Quelle: Meet-the-Expert, München, 21. Januar 2010 (Veranstalter: Essex)

\section{Kurz notiert}

Vorsicht, Fälschung - Viele Männer mit erektiler Dysfunktion (ED) suchen außerhalb des Gesundheitssystems nach Therapieoptionen - und begeben sich dadurch doppelt in Gefahr: Zum einen sind Potenzmittel von unseriösen Internetanbietern häufig falsch deklariert und mit gefährlichen Nebenwirkungen behaftet. So wurden z. B. zwischen 2004 und Sommer 2009 weltweit 40 Millionen gefälschte Viagra ${ }^{\circledR}$ Tabletten sichergestellt. Zudem haben viele ED-Patienten Begleiterkrankungen wie eine KHK und sollte vor Anwendung eines Potenzmittels ärztlich untersucht werden. Pfizer

Antidementivum mit gemeinsamem Vertrieb - Sanofi-Aventis steigt in Vertrieb und Vermarktung des Cholinesterasehemmers Rivastigmin (Exelon ${ }^{\circledR}$ ) von Novartis mit ein. Rivastigmin ist zur Behandlung der Alzheimerdemenz im frühen bis mittleren Stadium indiziert und wird außer zur oralen Therapie als einziges Antidementivum auch als Pflaster angeboten. Sanofi-Aventis

\section{Zweiter Protonenpumpenhemmer} generisch $>$ Der Protonenpumpenhemmer (PPI) Pantoprazol ist jetzt auch rezeptfrei von Hexal erhältlich. Pantoprazol Hexal ${ }^{\circledR}$ bei Sodbrennen ist bioäquivalent zu Pantozol Control $^{\circledR}$ und zugelassen zur kurzfristigen Behandlung von Refluxsymptomen. Hexal hat bereits vor einem halben Jahr den PPI Omep Hexal ${ }^{\circledR}$ für die Selbstmedikation eingeführt.

Hexal 